\title{
Molecular Cloning and Structure Analysis Of MpYop1, a Membrane Protein in Monascus purpureus
}

\author{
Haoming $\mathrm{Li}^{\mathrm{a}}$, Lan Gao ${ }^{\mathrm{b}}$ \\ School of Biosciences and Biopharmaceutics, Guangdong Pharmaceutical University, Guangzhou \\ Higher Education Mega Center, Guangzhou 510006, Peoples Republic of China \\ ${ }^{a}$ Corresponding author, Lihaoming@gdpu.edu.cn, bolgdpu@aliyun .com
}

Keywords: Membrane; Transmembrane helix; cloning; Monascus purpureus; endoplasmic reticulum.

Abstract. In all eukaryotes, the peripheral endoplasmic reticulum (ER) branches out of the nuclear envelope as a membrane network of interconnected tubules and membrane sheets with a single lumen. In yeast, Rtn1p and Yop1p are major, redundant components required to maintain the tubular ER under normal circumstances. In this work, we have investigated the homolog of the yeast Yop1 protein in Monascus purpureus. An M. purpureus cDNA library was constructed, and the MpYop1 cDNA was isolated from the cDNA library by random sequencing method. The cDNA was $1008 \mathrm{bp}$ in length, contains a predicted 516 bp ORF that encodes 171 amino acids, the gene was designated MpYop1. The deduced amino acid sequence showed homology to Yop1 from fungi. The TM topology model of MpYop1 was built, the secondary structure is similar to yeast Yop1p. MpYop1 contains four transmembrane $\alpha$-helices connected by a cytoplasmic loop and two extracellar loop, both the $\mathrm{N}$ and $\mathrm{C}$ termini are located in the cytoplasm side of ER membrane. According to functions of Yop1 in Yeast, we implicated that the MpYop1 would involved in stabilization of tubule ER.

\section{Introduction}

The endoplasmic reticulum (ER) is a continuous membrane system which has many different functions. These include the translocation of proteins across the ER membrane; the folding and modification of proteins in the ER lumen; the synthesis of phospholipids and steroids on the cytosolic side of the ER membrane; and the storage of calcium ions in the ER lumen and their regulated release into the cytosol[1-2]. ER is often tightly associated with almost every other membrane-bound compartment in the cell including the plasma membrane, nuclear envelope, mitochondria, golgi, vacuoles and peroxisomes. Interactions between these organelles have shown to be functionally important [3].

The ER consists of the nuclear envelope and a peripheral network of tubules and membrane sheets. Peripheral ER sheets with little curvature except at their edges and the nuclear envelope with low curvature except where the nuclear pores are inserted. The ER is composed of a network of "rough," ribosome-studded sheets and smooth tubules that are continuous with the nuclear envelope. These subdomains are plastic and are remodeled in response to the needs of the cell. During interphase, enhanced protein synthesis and secretion promote morphology rich in sheets and rough ER, whereas lipid and steroid synthesis and detoxification promote abundant tubular smooth ER [4].

The mechanism of ER network formation and maintenance is unclear. The possible model is based on mechanisms that generate or stabilize high curvature in membranes. Two protein families, the reticulons (Rtns) and DP1/Yop1 (Yip one partner), have been shown to shape the membrane bilayer of ER tubules in multiple eukaryotes, including animals, plants, and yeast [5-6]. Members of both families are ubiquitously expressed in all eukaryotic cells. In yeast, Rtn1p (one of reticulons) and Yop1p are most abundant membrane proteins [7]. Yeast lacking both reticulons and Yop1p had disrupted ER under normal growth conditions [5]. Conversely, the overexpression of certain isoforms results in long, unbranched tubules. Reticulons and DP1/Yop1p almost exclusive localized to the tubular ER, these two protein families share little overall sequence homology, but both have a conserved domain containing two elongated hydrophobic segments ( $\sim 30-35$ residues) that sit in the 
membrane as hairpins $[5,8]$. It is identified that the DP1/Yop1p may generate sheets by stabilizing the high curvature of edges [9]. And there are reports that reticulons and Yop1/DP1 play a role in the early steps of de novo NPC (the nuclear pore complexes) assembly and SPB (the spindle pole bodies) function in S. cerevisiae [10].

The 3D analysis of yeast ER reveals that during six sequential stages of budding, the peripheral ER contains cytoplasmic cisternae, many tubules, and a large plasma membrane (PM)-associated ER domain that consists of both tubules and fenestrated sheets. Mother cell lacking Rtn/Yop1 were depleted of tubules and all other peripheral ER regions of membrane curvature, but does not prevent ER tubules being pulled out of the mother cisternae into the bud, it is consistent with a model that Rtns/Yop1 stabilize and maintain regions of membrane curvature rather than generate them in yeast [11-12].

\section{Materials and Methods}

Strains, and Growth Conditions M. purpureus strain CICC 5031 was purchased from china center of industrial culture collection. The stock culture was maintained on PDA (potato dextrose agar) slants. Spores were prepared by growth on these slants for 7 days at $30^{\circ} \mathrm{C}$, washed and harvested with sterile water. A suspension of spores was used to inoculate a $250 \mathrm{ml}$ flask containing $40 \mathrm{ml}$ liquid fermentation medium PD (potato dextrose broth), for cDNA library construction, the M. purpureuswas incubated at $30^{\circ} \mathrm{C}$ at $130 \mathrm{rpm}$ for 5 days. The mycelium samples were collected and washed with sterile water, frozen in liquid nitrogen, and then were stored at $-80^{\circ} \mathrm{C}$ until required.

CDNA Library Construction, ESTs Sequencing and Cloning of MpYop1 Total RNA was extracted from frozen mycelium $\left(40 \mathrm{ml}\right.$ culture which incubated at $30^{\circ} \mathrm{C}, 130 \mathrm{rpm}$ for 5 days) using TRIzol ${ }^{\circledR}$ reagent (Invitrongen, USA), following the instructions of manufacturer. From total RNA, the cDNA library construction and amplification were performed following the users manual of the Creator TM SMARTTM cDNA Library construction Kit (Clontech, USA). The SMART cDNAs were ligated into SfiI-digested pDNR-LIB vector and transformed into Escherichia coli strain DH5 $\alpha$. Colonies were randomly picked, inoculate each colony to separated PCR reaction solutions. The colony was lysised by heating the mixed solutions at $95^{\circ} \mathrm{C}$ in a PTC-200 Thermocycler (MJ Research, USA) for 5 min.

After then, went to PCR amplification procedure with M13 primers provided by the CreatorTM SMARTTM cDNA Library construction Kit. The amplified PCR products (ESTs, expressed sequence tags) were analyzed by $1.2 \%$ agarose gel electrophoresis. When the amplified cDNA fragments were longer than $1000 \mathrm{bp}$, incubated the isolated colonies and sequenced the ESTs. After sequencing and analysis, the colony containing the predicted pDNR-LIB-MpYop1 was isolated.

\section{Results and Discussion}

To identify gene encoding MpYop1 protein in M. purpureus, a cDNA library of mycelium was constructed and screened with primers M13 (see method). The inserts of about 200 clones were sequenced and analyzed. The full-length MpYop1 cDNA was then obtained (Genbank accession No. GU797552). The cDNA was 1008 bp in length, contains a predicted 516 bp ORF that encodes 171 amino acids, a 96 bp non-coding region at $5^{\prime}$ end and a 396 bp of non-coding region flank at $3^{\prime}$ end. The predicted amino acid sequence of MpYop1 specifies a polypeptide with a molecular mass of $19.15 \mathrm{kDa}$.

Protein sequence alignment revealed that the deduced MpYop1 protein is highly homologous to other known Yoplps of Eurotiomycetes; and the sequence homology is relatively lower with yeast (Dothideomycetes) (Fig.1). MpYop1 had an 83.04\% identity with Yop1 in Talaromyces marneffei, $81.87 \%$ identity with Penicillium roqueforti, $80.70 \%$ identity with Aspergillus fumigatus, Neosartorya udagawae and Byssochlamys spectabilis, $80.12 \%$ identity with Coccidioides posadasii, 79.53\% identity with Aspergillus ruber, 76.44\% identity with Emmonsia crescens, $75.72 \%$ identity with Histoplasma capsulatum and $36.11 \%$ identity with Saccharomyces cerevisiae. The primary 
sequence of MpYop1p is predicted to have 2 long hydrophobic and 4 short alpha helices similar to yeast.

A phylogenetic tree was constructed for 20 sequences (Fig.2). Sequences were aligned using clustalW2. Phylogenetic trees were generated using MEGA4. The tree was built using neighbor-joining algorithm approach. From the tree in Fig.2, it's showed that MpYop1 protein is closer to Yop1s from fungi.

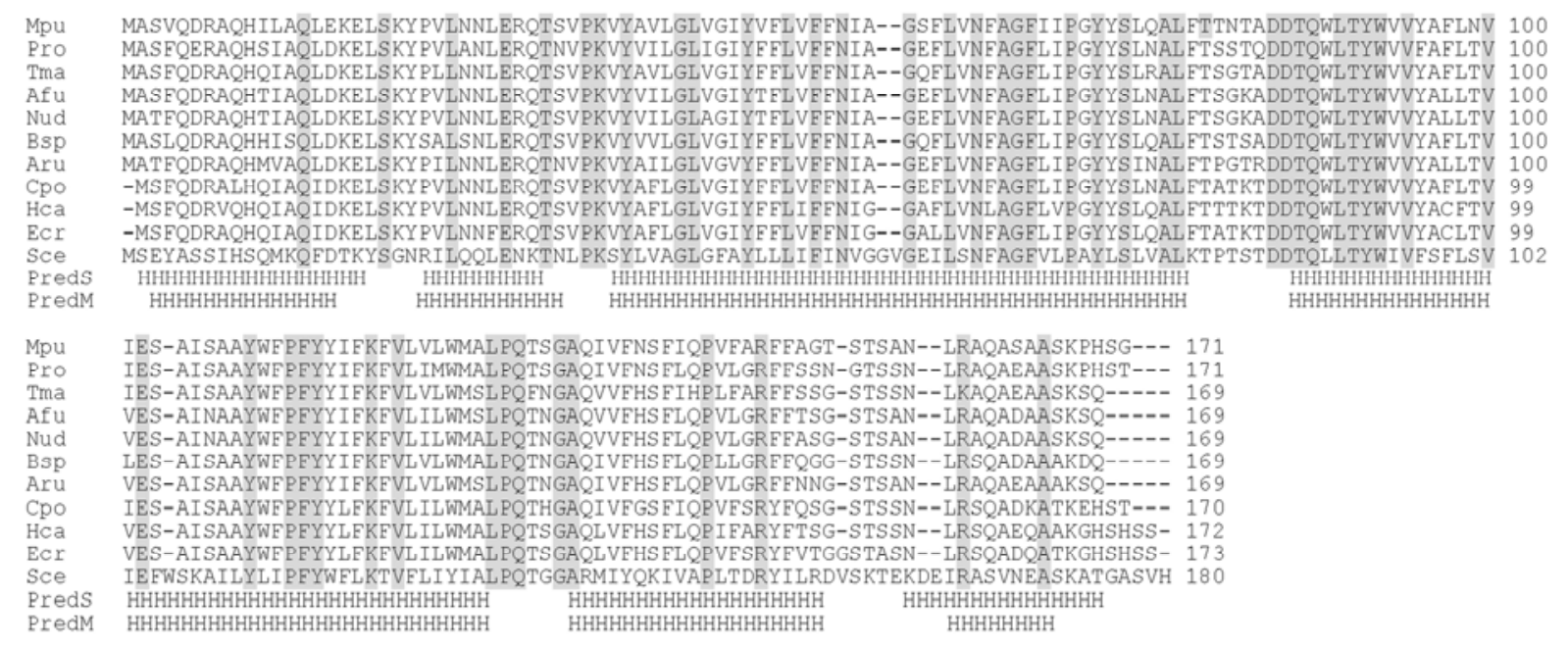

Fig. 1.Alignment of MpYop1 with 10 protein sequences from fungi. The abbreviation and Genbank accession numbers are: Mpu (Monascus purpureus, AIX10960), Pro (Penicillium roqueforti CDM29347), Tma (Talaromyces marneffei, XP_002147991), Afu (Aspergillus fumigatus, XP_754001), Nud (Neosartorya udagawae, GAO82259), Bsp (Byssochlamys spectabilis, GAD98562), Aru (Aspergillus ruber, EYE93430), Cpo (Coccidioides posadasii, XP_003069377), Hca (Histoplasma capsulatum, XP_001536604), Ecr (Emmonsia crescens, KKZ65202) and Sce (Saccharomyces cerevisiae, EWH15508). PredS and PredM, predict secondary structure of Sce and Mpu by PSIPRED v3.3, alpha helices are marked with H. Identical residues are shaded.

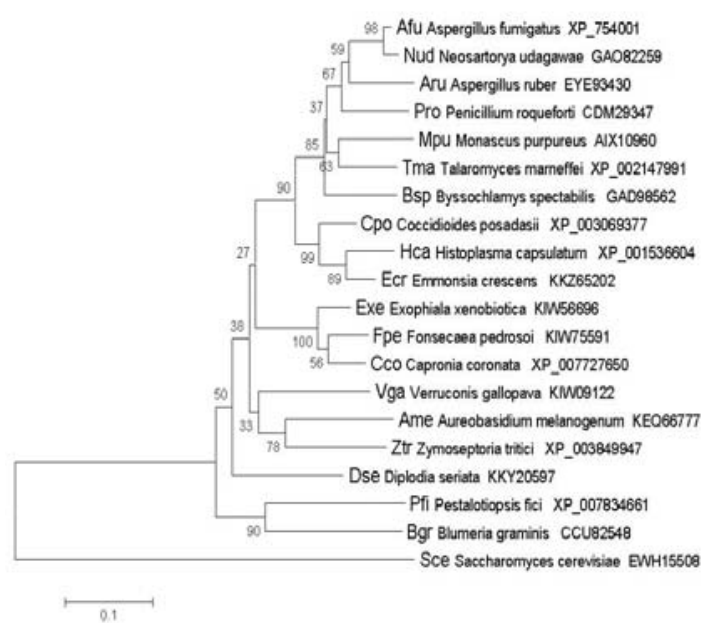

Fig. 2 Phylogeny of 20 Yop1 proteins of fungi. The Genbank accession numbers are indicated.

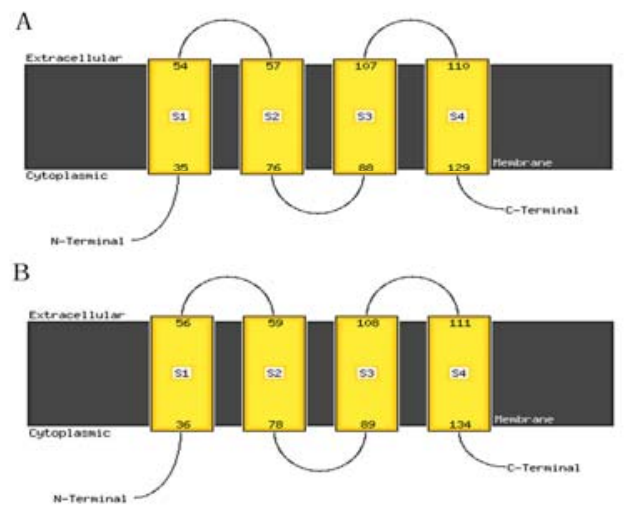

Fig. 3 TM topology prediction of MpYop1 and ScYop1p by the PSIPRED protein sequence analysis workbench. (A) MpYop1; (B) ScYop1p.

A major difference between ER tubules and the sheets of the nuclear envelope and peripheral ER is that tubules have a high curvature in cross-section. The reticulons and DP1/Yop $1 p$ may function to stabilize such high curvature membranes. In the absence of Yop1p, ER tubules can be maintained by Rtn1p alone, probably because Rtn1p can form oligomers on its own. The reticulons and DP1/Yop1p may be similar to caveolin, and integral membrane protein that forms oligomers and is abundant in and required for the formation of curved invaginations of the plasma membrane, called caveoli [13]. The reticulons do not share any primary sequence homology with members of the DP1/Yop1p family. 
However, both families have a conserved domain of 200 amino acids containing two long hydrophobic segments. Each hydrophobic segment seems to form a hairpin within the lipid bilayer so that all hydrophilic segments are found in the cytoplasm. The hydrophobic hairpins are proposed to form a "wedge" shape within the lipid bilayer, which would increase the surface area of the outer membrane leaflet to create the high curvature of ER tubules seen in their cross-section. [14-15].

The predicted TM topology and helix packing for MpYop1 and yeast Yop1 by PSIPRED are shown in Fig.3. MpYop1 has 4 transmembrane helixes, the first two are belong to the first long hydrophobic alpha helices, from K36 to A56 and from F59 to F78; the second two are belong to the second long hydrophobic alpha helices, from L89-A108 and from F111-Q134. Each two hydrophobic segment forms a hairpin within the membrane. Four transmembrane $\alpha$-helices connected by a cytoplasmic loop and two extracellar loop; both the $\mathrm{N}$ and $\mathrm{C}$ termini are located in the cytoplasm side of ER membrane.

\section{Conclusion}

The TM topology of MpYop1 is similar to yeast Yop1. Suggest that the MpYop1 may have the same function with yeast Yop1. MpYop1 would be a member of curvature-stabilizing proteins along with reticulons.

\section{Acknowledgements}

This work was partially financially supported by the Guangdong Sci. \& Tech. Program (2013B010404046).

\section{References}

[1] A.R. English, N.Zurek and G.K. Voeltz: Curr. Opin. Cell Biol, Vol. 21(2009), p. 596-602.

[2] C.R. McMaster: Biochem. Cell Biol, Vol. 79 (2001), p. 681-692.

[3] S. Böckler and B. Westermann: Dev. Cell, Vol. 28 (2014), p. 450-458.

[4] M. Puhka, M. Joensuu, H. Vihinen et al: Mol. Biol. Cell, Vol. 23(2012), p. 2424-2432.

[5] G.K. Voeltz, W.A, Prinz, Y. Shibata et al: Cell, Vol. 124(2006), p. 573-586.

[6] C.N. Chen, H.R. Chen, S.Y. Yeh et al: Plant Physiol, Vol. 149(2009), p. 1679-1689.

[7] S. Ghaemmaghami, W.K. Huh, K. Bower et al: Nature, Vol. 425(2003), p. 737-741.

[8] S.H. Park and C. Blackstone: EMBO Rep, Vol. 11(2010), p. 515-521.

[9] Y. Shibata, T. Shemesh, W.A. Prinz et al: Cell, Vol. 143(2010), p. 774-788.

[10]A.K. Casey, T.R. Dawson, J. Chen et al: Genetics, Vol. 192(2012), p.441-455.

[11]M. West, N. Zurek, A. Hoenger et al: J. Cell Biol, Vol. 193(2011), p. 333-346.

[12]L. Costantini and E. Snapp: Curr. Protoc. Cell Biol, Vol. 60(2013), p. Unit 21.7.

[13]R.G. Parton and A.A.Richards: Traffic, Vol. 4(2003), p. 724-738.

[14]Y. Shibata, C. Voss, J.M. Rist et al: J Biol Chem, Vol. 283(2008), p. 18892-904.

[15]P. Ngamskulrungroj, Y. Chang, B. Hansen et al: FEMS Yeast Res, Vol. 12(2012), p. 748-754. 